\section{CPC-089 MULTIDISCIPLINARY COLLABORATION IN THE TREATMENT OF PAEDIATRIC HEMATOPOIETIC TRANSPLANT REJECTION WITH ALLOGENEIC MESENCHYMAL CELLS. A CASE REPORT}

doi:10.1136/ejhpharm-2013-000276.546

${ }^{1} \mathrm{R}$ Garcia Ramos, ${ }^{1} \mathrm{M}$ Gonzalez Barcia, ${ }^{1} \mathrm{M}$ Rodriguez Prada, ${ }^{2} \mathrm{~A}$ Campos Pena, ${ }^{2} \mathrm{M}$ Villamayor Alvarez, ${ }^{3} \mathrm{JM}$ Couselo Sanchez. 'Complexo Hospitalario Universitario de Santiago de Compostela, Pharmacy, Santiago de Compostela, Spain; ${ }^{2}$ Complexo Hospitalario Universitario de Santiago de Compostela, Hematology, Santiago de Compostela, Spain; ${ }^{3}$ Complexo Hospitalario Universitario de Santiago de Compostela, Pediatrics, Santiago de Compostela, Spain

Background Advanced treatments represent a source of hope for rare diseases. However, they are complex as they require the participation of several professionals and experience is necessary for optimal use.

Purpose To describe the outcome and collaborative multidisciplinary process undertaken for the appropriate use of allogeneic mesenchymal cells (AMC) in the treatment of graft versus host disease (GVHD) developed by a paediatric patient after hematopoietic stem cell transplantation.

Materials and Methods Retrospective study of clinical outcomes, steps taken and requirements for the preparation of AMC (Prochy$\mathrm{mal}$ ). The case involved a 2-year-old paediatric patient with steroidrefractory severe GVHD with severe gastrointestinal manifestations. The treatment involved the administration of two doses per week for a total period of 4 weeks. If the patient responds completely or not at all, the treatment is completed, if there is a partial response the treatment can be completed plus an additional weekly dose for 4 extra weeks.

Results There was cooperation between the Paediatrics, Haematology and Pharmacy Services. A protocol was developed for use based on the instructions provided by the supplier. Pharmacy processed the application as a compassionate use (expanded access clinical trial) with the agreement of the supplier and hospital management. Haematology built on its expertise in handling blood cells to ensure storage $\left(-135^{\circ} \mathrm{C}\right)$ and initially collaborated with Pharmacy in the preparation of the doses: controlled defrosting, bottling and packaging in aseptic conditions. The treatment resulted in a partial response at completion so an additional cycle was administered. No adverse reactions to AMC were observed.

Conclusions Interdisciplinary collaboration through the optimization of hospital resources and the rapid training of participating staff allowed the administration of a new and urgent treatment of advanced treatment, allogeneic mesenchymal cells. Tolerance was good and the response to treatment was initially favourable.

No conflict of interest.

\section{CPC-090 MUPIROCIN RESISTANT METICILLIN-RESISTANT STAPHYLOCOCCUS AUREUS (MRSA) - DO PATIENTS GET THE CORRECT DECOLONISATION AFTER SCREENING?}

doi:10.1136/ejhpharm-2013-000276.547

'P Howard, ${ }^{2} \mathrm{~K}$ Morris. 'Leeds Teaching Hospitals NHS Trust, Pharmacy, Leeds, UK; 'Leeds Teaching Hospitals NHS Trust, Microbiology, Leeds, UK

Background MRSA screening has been mandatory in England for two years. Mupirocin is used routinely for MRSA-positive patients but there is some resistance.

Purpose A prospective audit was undertaken of all mupirocinresistant MRSA screens to see if patients were put on the correct treatment.

Materials and Methods From October 2011, all in-patients with high level mupirocin or neomycin MRSA resistance were followed up by a pharmacist. Patients on ineffective decolonisation regimes were changed to the correct regime, and ward staff educated Results to March 2012 were shared, and then monthly thereafter. Education was delivered at speciality and ward level. Any subsequent failures were reported as clinical incidents.

Results The percentage of patients that were MRSA positive on screening on admission into hospital or at pre-elective screening remained stable at $2.3 \%$ during both periods. Worryingly high-level mupirocin resistance had increased from $12.2 \%$ to $19.7 \%$. It had doubled to $24 \%$ by June! Despite audit, education and feedback, the proportion of patients with known MRSA on admission and those still in hospital when the result from the admission screen was released, on the correct decolonisation regime, got worse. There has been no improvement using senior staff or ward infection control link nurses to rebrief their staff on the documented procedure.

Conclusions MRSA carriage on screening is low. Current systems appear too complex despite multiple interventions. As a failsafe, these patients should be followed up. Posters and screensavers have since been introduced. The prospective audit continues. All centres should review their current practise to ensure patients get prescribed effective MRSA decolonisation.

\section{Asbtract CPC-090 Table 1}

\begin{tabular}{lll}
\hline & Oct 2011-Mar 2012 & Apr-Jun 2012 \\
\hline Screens & 49177 & 17926 \\
MRSA positive & $2.3 \%$ & $2.3 \%$ \\
High level mupirocin resistance & $12.2 \%$ & $19.7 \%$ \\
And also neomycin resistant & $8.6 \%$ & $4.6 \%$ \\
Correct on admission (from previous screen results) & $23 \%$ & $12 \%$ \\
Correct after current admission screen result released & $40 \%$ & $27 \%$ \\
\hline
\end{tabular}

No conflict of interest.

\section{CPC-091 NATALIZUMAB IN CYPRIOT PATIENTS WITH RELAPSING REMITTING MULTIPLE SCLEROSIS: THREE YEAR DATA ON SAFETY, EFFICACY AND FREQUENCY OF ANTI-JC VIRUS ANTIBODIES}

doi:10.1136/ejhpharm-2013-000276.548

E Kkolou, E Gaglia, J Toufexis, M Pantzaris. The Cyprus Institute of Neurology and Genetics, Clinical Neurosciences, Nicosia, Cyprus

Background Natalizumab (NAT) is a recombinant humanised anti- $\alpha 4$-integrin antibody used in treating Relapsing Remitting (RR) Multiple Sclerosis (MS).

Purpose To evaluate the long-term safety and efficacy of NAT in Cypriot patients, to assess the frequency of anti-JC Virus (JCV) antibodies and implement a strategy for the prevention of PML

Materials and Methods Twenty-two patients were studied prospectively for 3 years.

The patients received $300 \mathrm{mg}$ of NAT intravenously every 4 weeks. MRI examinations were performed at study entry and 12-24 months after the start of treatment. JCV antibody testing was performed after two years of treatment.

Results Six patients (27.3\%) discontinued the study due to: Severe allergic reaction (9\%), generalised atony, fatigue and weakness (4\%), recurring herpes infection (4\%), family planning (4\%) and presence of anti-JCV antibodies (anti-JCV positive) due to previous immunosuppressive therapy (4\%).

Most frequently reported side effects were: cardiovascular (41\%), general (41\%), laboratory abnormalities (41\%), gastrointestinal $(23 \%)$, neurological (18\%), allergic reactions (18\%) and depression (14\%).

After three years of NAT treatment, a $55.2 \%$ decrease from the baseline mean annual relapse rate was observed, as well as improvement of 0.3 points on the mean Expanded Disability Status Scale (EDSS) Score. 
$87.5 \%$ of the patients completing the study had repeat MRI scans. Of those, $85.7 \%$ were found to have no new or gadoliniumenhancing lesions.

JC Virus antibody testing was performed after two years of NAT. Of the thirteen samples, eight $(61.5 \%)$ tested positive. Two of those (25\%) discontinued NAT due to previous IV mitoxantrone treatment. The remaining patients continued treatment under close supervision by the attending neurologist.

No cases of Progressive Multifocal Leukoencephalopathy were reported.

Conclusions Long-term therapy with natalizumab proved to be safe and effective in our population. Strict follow-up criteria were implemented for JCV antibody-positive patients remaining on treatment with natalizumab for more than two years.

No conflict of interest.

\section{CPC-092 NEUROPSYCHOLOGY OF SAUDI COLON CANCER PATIENTS}

doi:10.1136/ejhpharm-2013-000276.549

'N Abanmy, ${ }^{2 P}$ Gard, ${ }^{2}$ A Macadam, '0 Alomran. 'King Saud University, Clinical Pharmacy, Riyadh, Saudi Arabia; 'University of Brighton, Pharmacology and Therapeutics, Brighton, UK

Background Colorectal cancer is a common disease and its prevalence is second to that of breast cancer worldwide. In Saudi Arabia the disease is ranked second after breast cancer and accounts for $8.5 \%$ of all tumours. Evaluation of Quality of life (QOL), anxiety and depression of such patients, as well as neurocognitive properties, is important to assess the impact of both malignancy and/or exposure to treatments including chemotherapy and surgery.

Purpose To assess the neuropsychology of a group of Saudi colon cancer patients 6 months after treatment was completed.

Materials and Methods Patients (18- 60 years) were recruited from the oncology clinic at King Khaled University Hospital (KKUH) at Riyadh, the capital city of Saudi Arabia. Exclusion criteria included smoking, psychiatric or cerebrovascular disease, sensory impairment, abnormal electrolytes, anaemia or uncontrolled blood pressure. Healthy volunteers were randomly recruited from the same hospital, however the availability of matched age controls was difficult. Cognition was assessed using the Rey Auditory-Verbal Test RAVLT (learning \& memory); the Rey-Osterrieth complex figure (RCF, visuo-spatial organisation and visuo-spatial memory); semantic verbal fluency (executive function); letter cancellation (attention); digit-symbol (sustained attention, visual searching, visual sequencing). The Arabic version of 36-item Short-Form Health Survey SF-36 and the Hospital Anxiety and Depression Scale (HADS) were also used to assess QOL, anxiety, and depression, respectively.

Results A total of 32 colon cancer patients in remission were recruited, their mean age was 44.8 years. 23 of them were males $(71.9 \%)$, while their mean years of education was $13.1 \pm 4.06$ years. Healthy controls $(n=36)$, were significantly younger than the patients (34 Years) $(\mathrm{t}(66)=-4.2, \mathrm{P}<0.05)$. There were no differences between the groups in terms of QOL, anxiety, depression, attention, executive function, oral and visuospatial memory. Healthy controls had significantly better RCF recall task $(\mathrm{t}(67)=2.61, \mathrm{p}<0.01)$ and delayed recall task $(\mathrm{t}(67)=3.16$, $\mathrm{p}=0.002)$ than colon cancer patients.

Conclusions This study indicates that neither colon cancer, nor its treatment, has any significant impact on the psychological wellbeing of the patients in comparison to healthy controls. The significant differences in recall may reflect the differences in age between the groups.

No conflict of interest.

\section{CPC-093 NOSOCOMIAL INFECTIONS IN A COHORT OF EXTRA-CORPOREAL LIFE SUPPORT PATIENTS}

doi:10.1136/ejhpharm-2013-000276.550

'D Lenehan, 'N Scanlon, ${ }^{2}$ Conrick-Martin, ${ }^{3} \mathrm{~J}$ O'Gorman, ${ }^{2} \mathrm{D}$ Oshodi, ${ }^{2} \mathrm{~S}$ O'Brien, ${ }^{3} \mathrm{M}$ Hannan, ${ }^{3} \mathrm{M}$ Lynch, ${ }^{2} \mathrm{E}$ Carton, ${ }^{1} \mathrm{C}$ Meegan. ${ }^{\prime} \mathrm{MMUH}$, Pharmacy, Dublin, Ireland (Rep.); ' ${ }^{2} \mathrm{MMUH}$, Intensive Care Unit, Dublin, Ireland (Rep.); ${ }^{3} \mathrm{MMUH}$, Microbiology, Dublin, Ireland (Rep.)

Background Extra-Corporeal Life Support (ECLS) is a method of life support used to treat patients with severe respiratory and/or cardiac failure refractory to conventional modes of treatment. Nosocomial infections in these patients are associated with increased morbidity and mortality along with increased lengths of intensive care unit (ICU) and hospital stay. No international best-practise consensus guidelines exist for treatment and/or prophylaxis of infections in this patient group.

Purpose To examine the rate of nosocomial infection in $\mathrm{MMUH}$ ECLS patients as well as the consumption of antimicrobials in the treatment and prevention of these infections.

Materials and Methods In a retrospective cohort study, the pharmacy records from a daily multi-disciplinary microbiology round reviewed all patients who are on ECLS. The use of prophylactic and therapeutic antimicrobials in these patients was assessed as well as the background ICU bloodstream infection rate.

Results Data analysis yielded a total of 17 patients over a two-year period, with a total of 444 ECLS days. In total, there were 17 infections in this cohort including $4(24 \%)$ blood-stream infections (yielding a rate of 9.0 per 1000 ECLS days). The first four ECLS patients received antibacterial (vancomycin) and antifungal (caspofungin) prophylaxis for the duration of ECLS, whereas the later cohort of 13 did not. In the cohort of patients who received prophylactic antimicrobials, defined daily doses (DDDs) per 100 ECLS days for vancomycin and meropenem were 49.54 and 49.63 respectively. For the non-prophylaxis cohort this was 25.31 and 37.73 respectively.

Conclusions The infection rate in this cohort was low. In particular, the bloodstream infection rate compared favourably with previously published rates, and was comparable with the 'background' bloodstream infection rate of the ICU population as a whole. Antimicrobial use in ECLS patients was high relative to overall ICU antimicrobial use.

No conflict of interest.

\section{CPC-094 NUTRITIONAL STATUS OF HOSPITALISED PATIENTS WITH HEAD AND NECK CANCER}

doi:10.1136/ejhpharm-2013-000276.551

C Vázquez López, M Suárez Santamaría, M Alvarez Payero, D Pérez Parente, MS Ucha Samartín, G Piñeiro Corrales. Meixoeiro Hospital (CHUVI), Pharmacy, Vigo, Spain

Background Malnutrition is common in hospitalised patients with head and neck carcinoma.

Purpose The aim of this study was to analyse the causes of hospitalisation of patients with head and neck cancer and to evaluate the nutritional status; type and route of nutrition therapy during hospitalisation and at discharge.

Materials and Methods Retrospective study of patients with head and neck cancer, between October 2011 and March 2012 at 420-beds hospital.

We examined demographic data, cause of admission to hospital, type and location of tumour, nutritional status before admission and at discharge by CONUT ${ }^{\circledR}$ (system for early detection and monitoring of clinical undernutrition based on biochemical parametres and immune indicator) and type of nutritional therapy. We used the data source as medical record (IANUS ${ }^{\circledR}$ ). 